\title{
Distributed Three-Stage Concatenated Irregular Convolutional, Unity-Rate and Space-Time Trellis Coding for Single-Antenna Aided Cooperative Communications
}

\author{
Hung Viet Nguyen, Soon Xin Ng, and Lajos Hanzo \\ School of ECS, University of Southampton, SO17 1BJ, United Kingdom. \\ Tel: +44-23-8059 3125, Fax: +44-23-8059 4508 \\ Email: $\{$ hvn08r,sxn,lh\}@ecs.soton.ac.uk, http://www-mobile.ecs.soton.ac.uk
}

\begin{abstract}
In this contribution, we propose a Distributed Concatenated Irregular Convolutional Coded Unity Rate Coded and Space Time Trellis Coded (DC-IrCC-URC-STTC) scheme for cooperative communications employing multiple single-antenna relays. Each coding arrangement is designed for efficient decoding convergence by employing non-binary Extrinsic Information Transfer (EXIT) charts. The source node transmits two-stage IrCC-URC encoded symbols to two relays during the first transmission period. Then each relay performs two-stage URC-IrCC decoding and re-encodes the information bits using the three-stage concatenated coding scheme IrCC-URC-STTC. However, only the signals of one predefined STTC output are transmitted from each relay to the destination node during the second transmission period. At the destination, the received signals are treated as if they arrived from one transmitter employing STTC using two co-located transmit antennas. The simulation results show that the Monte-Carlo based performance of the DC-IrCC-URC-STTC scheme exactly matches the EXIT-chart analysis. It also performs closely to its idealised counterpart that assumes perfect decoding at the relays when an EXIT-chart based optimal algorithm is applied for selecting relays.
\end{abstract}

\section{INTRODUCTION}

The ultimate aim of designing a wireless communication system is to provide reliable high data rate links. Multi-Input Multi-Output (MIMO) systems provide a linearly increasing capacity as a function of the transmit power, provided the extra power is assigned to additional antennas [1]. Space Time Trellis Codes (STTCs) [2] and Space Time Block Codes (STBCs) [3], which are joint coding and transmit-receive diversity aided MIMO systems, constitute efficient techniques of communicating over fading channels [4]. However, it is impractical to allocate multiple antennas to a shirt-pocketsized mobile unit. Cooperative communication systems, which have recently attracted substantial research efforts [5]-[9], are capable of creating a Virtual MIMO (VMIMO) from multiple single-antenna relays. Depending on the type of the relays, the set of algorithms developed for cooperative communication can be categorised into two main groups, namely Amplify-and-Forward (AF) as well as Decode-and-Forward (DF) techniques [5]-[9]. Extrinsic Information Transfer (EXIT) charts have been introduced as an effective tool for analysing the convergence properties of iterative decoding aided concatenated coding schemes [10]. As an advantage, this can be achieved without performing time-consuming Monte-Carlo simulations. EXIT charts can be used for finding powerful codes exhibiting guaranteed convergence for a given channel condition [11]. Specifically, nearcapacity codes have been successfully designed by applying an EXITchart-based technique in [12]-[14].

Tuchler and Hagenauer [12], [15] proposed the employment of IRregular Convolutional Codes (IRCCs) in serial concatenated schemes, which are constituted by a family of convolutional codes having different rates, in order to design a near-capacity system. They were specifically designed with the aid of EXIT charts to improve

The financial support of the Vietnamese International Education Development (VIED) fund and the EU under the auspices of the OPTIMIX project as well as of the EPSRC, UK is gratefully acknowledged. the convergence behaviour of iteratively decoded systems. Each component code of the IRCCs encodes an appropriately selected fraction of the input bit stream. The appropriate fractions may be selected with the aid of EXIT-chart analysis in order to shape the inverted EXIT curve of the composite IrCC for ensuring that it matches the EXIT curve of the so-called inner decoder constituted by the detector. In this manner, an open EXIT-chart tunnel can be created at low Signal to Noise Ratio (SNR) values, which implies approaching the channel's capacity bound [16].

As a further advance, it was shown in [17], [18] that a recursive Unity-Rate Code (URC) should be employed as an intermediate code in order to improve the attainable decoding convergence. A URC can be used as a precoder for creating an inner code component having Infinite Impulse Response (IIR) in order to reach the $(1,1)$ point in the EXIT chart and hence to achieve an infinitesimally low Bit Error Ratio (BER) [14].

In a nutshell, a near-capacity three stage IrCC-URC-STBC scheme was designed in [11], which requires multiple antennas at both transmitter and receiver sides. We note, however that STTCs are capable of attaining coding gain in addition to their spatial diversity gain, while the STBCs of [11] can only achieve a spatial diversity gain [19] but no coding gain. Against this background, the novel contribution of this paper is that we amalgamate the ideas of distributed space time coding [7], [9] and near-capacity channel coding with the cooperative DF approach employing multiple singleantenna relays and use our IrCC-URC-STTC coding arrangement to design a near-capacity coding scheme for single-antenna aided cooperative relaying systems. In other words, our objective is to design a near-capacity coding scheme for optimising the overall system and to devise the algorithms for finding relays for cooperation in the most appropriate locations in order to provide the best possible performance.

All in all a distributed coding scheme based on the IrCC-URCSTTC arrangement can be employed for the sake of approaching the Discrete-input Continuous-output Memoryless Channel (DCMC) capacity of the single-antenna relay cooperative systems. The rest of the paper is structured as follows. Our system model is outlined in Section II. Details of the coding scheme and its coding/decoding processes are illustrated in Section III. The design aspects of evaluating relay channel's capacity, formulating near-capacity coding arrangements with the EXIT-chart aided method and choosing the appropriate relays using EXIT-chart based analyses are presented in Section IV. Our results are discussed in Section V, leading to our conclusions in the last section.

\section{SySTEM MODEL}

We consider the system depicted in Fig. 1, where the source node $(s)$, the two relay nodes $(r)$ and the destination node $(d)$ use a single antenna to transmit and receive signals. During the first transmission period, the $s$ node transmits its coded frame to the $r$ nodes. Then, after decoding and re-encoding the signals received from 


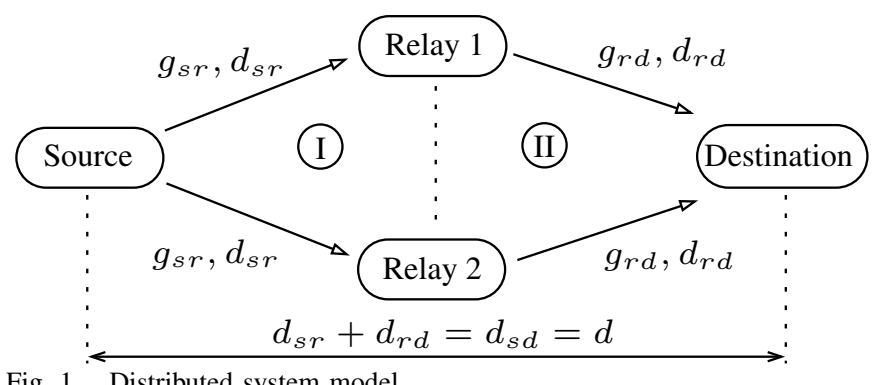

Fig. 1. Distributed system model

the $s$ node, the $r$ nodes transmit the resultant coded frame to the $d$ node during the second transmission period. At the destination, the signals received from the two single-antenna relays are combined, detected and decoded to recover the information transmitted from the $s$ node.

According to [5], [20], the power-gain (or geometrical gain), $g_{s r}$ expressed as a ratio or $G_{s r}(\mathrm{~dB})$, experienced by the source-to-relay link with respect to the source-to-destination link as a benefit of its reduced distance and path loss, can be computed as:

$$
g_{s r}=\left(\frac{d_{s d}}{d_{s r}}\right)^{2} .
$$

Similarly, the relay-to-destination link power-gain, $g_{r d}$ expressed as a ratio or $G_{r d}(\mathrm{~dB})$, with respect to the source-to-destination link can be formulated as:

$$
g_{r d}=\left(\frac{d_{s d}}{d_{r d}}\right)^{2} .
$$

Naturally, the power-gain at the source-to-destination link with respect to itself is unity, i.e. $g_{s d}=1$. Stipulating another assumption, namely that we have $d_{s d}=d_{s r}+d_{r d}=d$, and substituting it into (1) and (2), we accordingly infer that

$$
g_{r d}=\frac{g_{s r}}{\left(\sqrt{g_{s r}}-1\right)^{2}} .
$$

Let us define $S N R_{t}$ as the ratio of the power transmitted from the transmitter to the noise power encountered at the receiver. Following the approach in [5], [20], where the noise power, $N_{0}$, is assumed to be constant, then we have $S N R_{t \text { (source) }}=S N R_{t \text { (relay })}=S N R_{t}$, when the transmit power at the source equals that at the relays. Furthermore, we define $S N R_{r}$ as the ratio of the received power to the noise power at the receiver as $S N R_{r}=G+S N R_{t}$, where $G(\mathrm{~dB})$ is the power gain at the link. Accordingly, we obtain the corresponding equation as follows:

$$
S N R_{r(\text { relay })}-G_{s r}=S N R_{r(\text { dest. })}-G_{r d},
$$

where $S N R_{r(\text { relay })}$ and $S N R_{r(\text { dest.) }}$ are the receiver's SNRs at the relays and the destination, respectively.

Assuming that $i \in\left\{1, \ldots, M_{r}\right\}$ and $M_{r}$ is the number of relay nodes, while $k \in\left\{1, \ldots, N_{s}\right\}$ and $N_{s}$ is the number of symbols per frame transmitted from the $s$ node, the $k$ th received signal at the $i$ th $r$ node during the first transmission period can be formulated as:

$$
y_{r_{i}, k}=\sqrt{g_{s r_{i}}} h_{s r_{i}, k} x_{k}+n_{r_{i}, k},
$$

where $h_{s r_{i}, k}$ is the complex-valued Rayleigh fading coefficient between the $s$ node and the $i$ th $r$ node at instant $k$, while $n_{r_{i}, k}$ is the corresponding Additive White Gaussian Noise (AWGN) process having a variance of $N_{0} / 2$ per dimension. More explicitly, the $l$ th received symbol at the $d$ node during the second transmission period can be expressed as:

$$
y_{d, l}=\sqrt{g_{r_{1} d}} h_{r_{1} d, l} x_{r_{1}, l}+\sqrt{g_{r_{2} d}} h_{r_{2} d, l} x_{r_{2}, l}+n_{d, l},
$$

where $l \in\left\{1, \ldots, N_{r}\right\} ; N_{r}$ is the number of symbols per frame transmitted from the $r$ node; $h_{r_{i} d, l}$ is the complex-valued Rayleigh fading coefficient between the $i$ th $r$ node and the $d$ node at instant $l ; n_{d, l}$ is the AWGN having a variance of $N_{0} / 2$ per dimension.

\section{ENCODER AND DECODER}

In our Distributed Concatenated IrCC-URC-STTC (DC-IrCCURC-STTC) coding scheme depicted in Fig. 2, we use a 8PSKmodulated two-stage IrCC-URC encoder at the $s$ node. By contrast, at both the $r$ nodes and $d$ node, the three-stage IrCC-URC-STTC coding scheme using QPSK modulation is employed. Note that only the signals of one predefined STTC output at single-antenna $r$ nodes are transmitted. Thus, the signals received at the $d$ node are a combination of the signals received from two relays during the second transmission period. Then the signals received are treated as if they arrived from one relay transmitter equipped with two co-located transmit antennas.

Following Fig. 2, the information bit sequence $u$ is encoded by the IrCC encoder at the $s$ node to produce the coded sequence $c$, before passing $c$ through the interleaver. The interleaved bit sequence is encoded by the URC encoder and then modulated using 8PSK in order to form the transmitted symbol sequence $x$. The signals $y_{r 1}$ and $y_{r 2}$ received at both relays are iteratively decoded by the IrCC-URC decoders of Fig. 2 in order to estimate the original information bit sequences, $u_{1}$ and $u_{2}$, at $r$ node 1 and 2 , respectively. Then $u_{1}$ and $u_{2}$ are passed through the IrCC-URC-STTC encoding process with two interleavers between the IrCC-URC and URCSTTC arrangements. QPSK modulation later is employed. Note that the sequences $x_{r 1}$ and $x_{r 2}$ transmitted by each of the relays are created by choosing one predefined output of the two-antenna 4-state STTC encoder of [2]. The sequence $y_{d}$ received at the $d$ node is then subjected to the inverse process: $y_{d}$ is demodulated, and finally the resultant sequence is decoded by two iterative decoders, namely by the URC-STTC decoder and the IrCC-[amalgamated-URC-STTC] decoder. The decoded information bit sequence $u^{\prime}$ is compared with the original one to evaluate the BER.

The URC, IrCC and STTC component codes have the code rates of $1.0,0.5$, and 1.0, respectively. Thus, the code rates of the concatenated coding arrangements, namely of the IrCC-URC and IrCC-URC-STTC can be obtained according to $R_{I r C C-U R C}=R_{I r C C} \times R_{U R C}=0.5$ and $R_{I r C C-U R C-S T T C}=R_{I r C C} \times R_{U R C} \times R_{S T T C}=0.5$, respectively. The overall throughput of the proposed two-hop cooperative system can be calculated according to :

$$
\eta=\frac{N_{i}}{N_{s}+N_{r}}[b p s]
$$

where $N_{i}$ is the number of information bits transmitted within a duration of $\left(N_{s}+N_{r}\right)$ symbol periods. Since 8PSK is used for the source-relay transmission while QPSK is used for relay-destination transmission, we have $N_{s}=N_{i} /\left(R_{I r C C-U R C} \times \log _{2}(8)\right)=N_{i} \times$ $2 / 3$ and $N_{r}=N_{i} /\left(R_{I r C C-U R C-S T T C} \times \log _{2}(4)\right)=N_{i}$. From (7), we have $\eta=0.6$ [bps]. Accordingly, the SNR per bit is given by $E_{b} / N_{0}=S N R / \eta$.

\section{DESIGN AND ANALYSIS}

In this section, we first derive the relay channels' DCMC capacity for the DC-IrCC-URC-STTC scheme in Section IV-A. We then use the EXIT-chart aided method to design near-capacity coding arrangements for each transmission link of the DC-IrCC-URC-STTC system in Section IV-B. The results of our EXIT-chart based design are discussed in Section IV-C. 


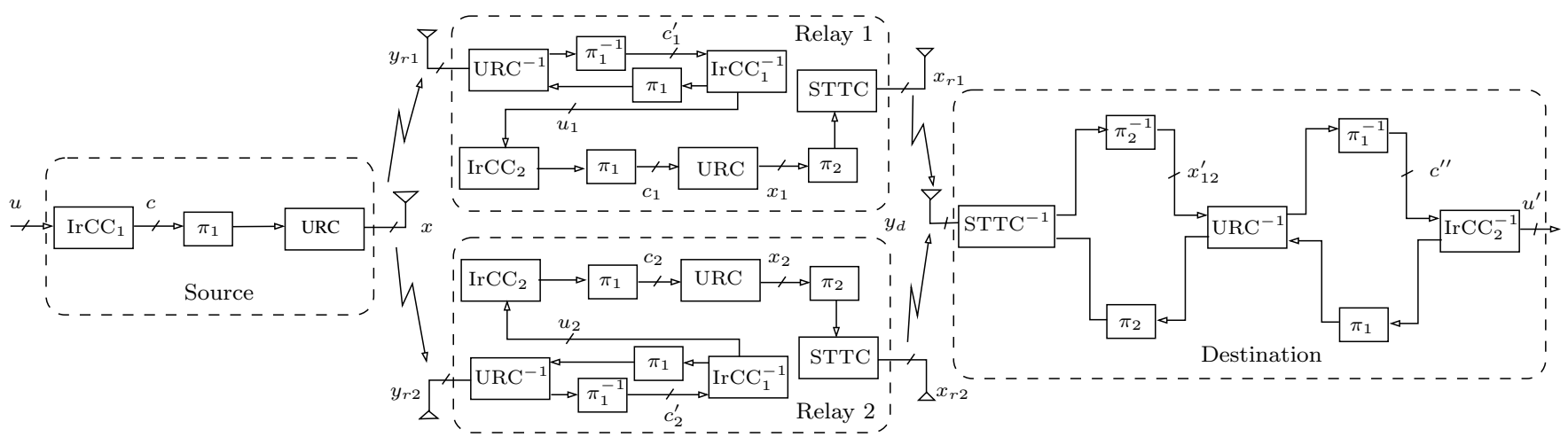

Fig. 2. The block diagram of the distributed concatenated IrCC-URC-STTC coding scheme

\section{A. Relay channel capacity}

According to [8], the achievable capacity of a full duplex relay aided Continuous-input Continuous-output Memoryless Channel (CCMC) can be calculated as follows:

$$
C_{C C M C}^{\text {relay }} \geq \max _{p\left(X_{1}, X_{2}\right)} \min \left\{I\left(X_{1} ; Y_{2} \mid X_{2}\right), I\left(X_{1}, X_{2} ; Y_{3}\right)\right\},
$$

where $X_{1}, X_{2}, Y_{2}$ and $Y_{3}$ are the signals transmitted from the $s$ node and $r$ node, as well as the signals received at the $r$ node and the $d$ node, respectively. Furthermore, $p\left(X_{1}, X_{2}\right)$ is the joint probability of signals transmitted from the $s$ and $r$ nodes. Applying the above general formulas to our half duplex DCMC system, assuming that the source-destination link is not available, while a pair of relays is viewed as a single equivalent relay having two transmit antennas, we can formulate the relay channel's achievable capacity as:

$$
\begin{array}{r}
C_{D C M C}^{\text {relay }} \geq \max _{p\left(X, X_{r}\right)} \min \left\{\frac{N_{s}}{N_{s}+N_{r}} \times E\left[I\left(X ; Y_{r}\right)\right],\right. \\
\left.\frac{N_{r}}{N_{s}+N_{r}} \times E\left[I\left(X_{r} ; Y_{d}\right)\right]\right\},
\end{array}
$$

where $X=\left[x_{1}, x_{2}, \ldots, x_{N_{s}}\right]$ represents the signals transmitted from the $s$ node with $x_{k}$ formulated in (5), while $X_{r}=$ $\left[x_{r_{1}, 1}, x_{r_{2}, 1}, \ldots, x_{r_{1}, N_{r}}, x_{r_{2}, N_{r}}\right]$ are the signals transmitted from the equivalent twin-antenna relay node with $x_{r_{i}, l}$ formulated in (6). Furthermore, $Y_{r}=Y_{r i}=\left[y_{r_{i}, 1}, \ldots, y_{r_{i}, N_{s}}\right]$ and $Y_{d}=\left[y_{d, 1}, \ldots, y_{d, N_{r}}\right]$ are the signals received at the equivalent relay node and the $d$ node with $y_{r_{i}, k}$ and $y_{d, l}$ calculated from (5) and (6), respectively.

\section{B. EXIT chart matching design}

We simplify the code design of the entire coding scheme by decomposing it into two separate EXIT curve matching scenarios: the first one, which is used for the source-relay link, is the IrCC outer code and the amalgamated URC-8PSK inner code; the second one, which is employed for the relay-destination link, is the IrCC outer code and the amalgamated URC-STTC-QPSK inner code.

In order to increase the achievable channel capacity of the almagamated URC-STTC-QPSK inner code and to approach that of the STTC-QPSK system, an iterative decoding process exchanging extrinsic information between the URC and STTC decoders should be implemented [21]. The simulation results seen in Fig. 3 show that once at least $I=3$ iterations were applied, the achievable channel capacities of the STTC-QPSK and URC-STTC-QPSK systems coincided.

The EXIT chart matching procedure is briefly summarised as follows:

Step1: Create the inner decoders' EXIT charts, namely that of the URC-8PSK arrangement for the source-relay link and that of the

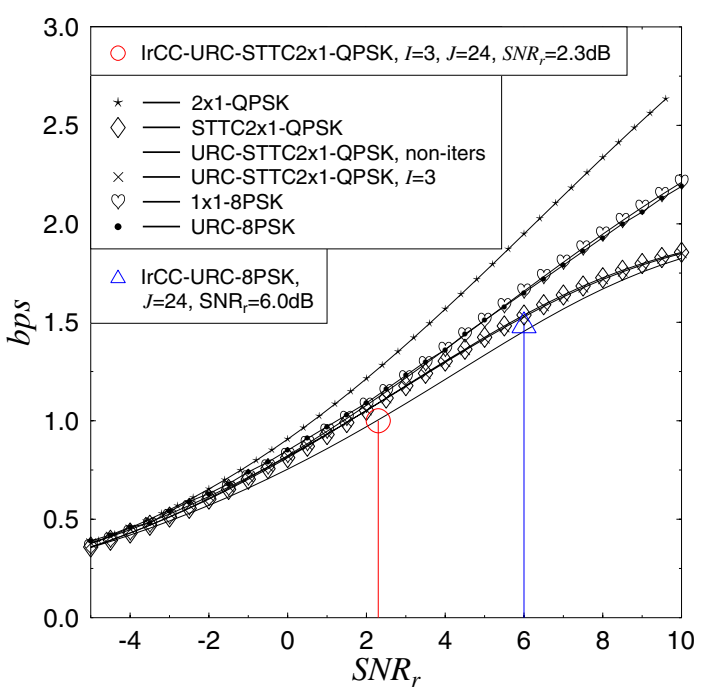

Fig. 3. Channel capacity comparison for the IrCC-URC-STTC2x1-QPSK and IrCC-URC-8PSK systems

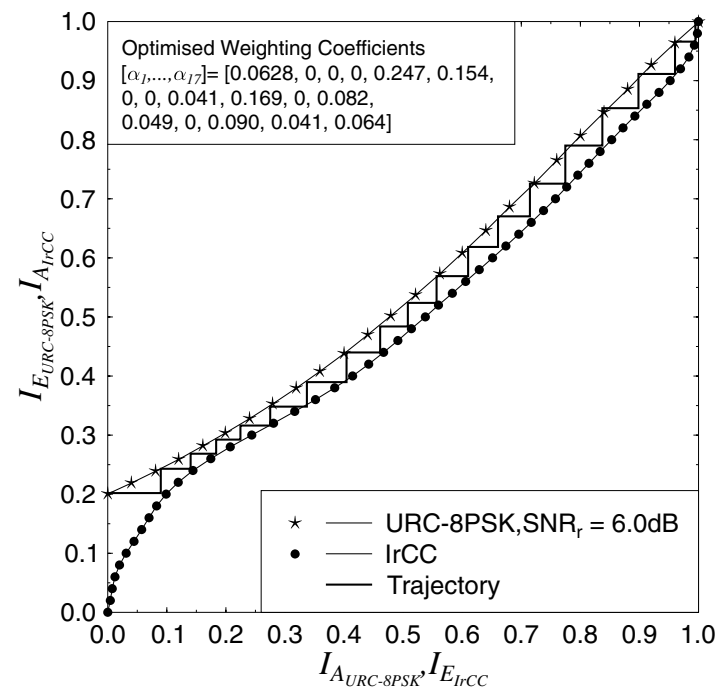

Fig. 4. The EXIT chart curves of the URC-8PSK and the IrCC for the source-relay link 
URC-STTC-QPSK scheme for the relay-destination link, for different receiver Signal to Noise Ratios $S N R_{r}$.

Step2: Fix the IrCC code rate to 0.5 and employ the EXIT curve matching algorithm of [12] to generate the optimised weighting coefficients $\alpha_{j}, j=1, \ldots, 17$, of the 17 component IrCC codes corresponding to the smallest $S N R_{r}$ that allows decoding convergence, where the decoding trajectory reaches the top-right corner of the corresponding EXIT charts. This implies that a near-capacity performance can be achieved.

Having implemented the steps mentioned above, we obtain the EXIT curves and the corresponding weighting coefficients $\alpha_{j}, j=$ $1, \ldots, 17$, for both scenarios, as shown in Fig. 4 and Fig. 5, respectively. The EXIT-chart results show that if having $J=24$ iterations were affordable, the trajectories would reach the $(1,1)$ point. Furthermore, the area property of EXIT-charts [13], [16] states that the area under the EXIT curve of an inner decoder component is approximately equal to the attainable channel capacity, provided that the channels' input symbols are equiprobable. By exploiting the area property of the EXIT-charts [13], [16], the achievable DCMC capacities of the STTC-QPSK, URC-STTC-QPSK, IrCCURC-STTC-QPSK, URC-8PSK, IrCC-URC-8PSK systems are quantified in Fig. 3. As seen from Fig. 3, Fig. 4 and Fig. 5, the IrCCURC-STTC-QPSK and IrCC-URC-8PSK scheme's capacity curves are only about $(2.3-1.6)=0.7 \mathrm{~dB}$ and $(6.0-5.0)=1.0 \mathrm{~dB}$ away from the STTC-based and URC-based DCMC capacity curves, respectively.

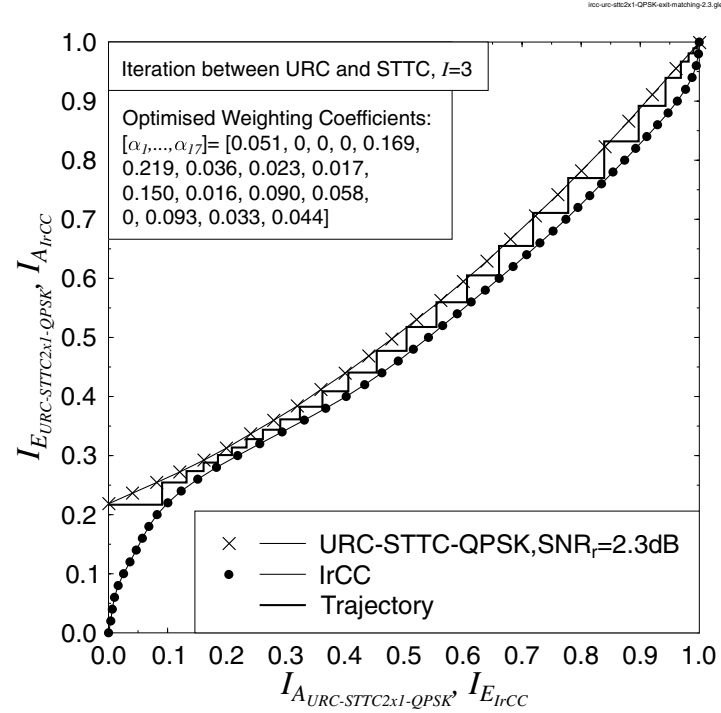

Fig. 5. The EXIT chart curves of the URC-STTC $2 \times 1-\mathrm{QPSK}$ and the IrCC for the relay-destination link

\section{Choosing Relays}

In order to allow our coding scheme to provide the best possible performance, two appropriate relays should be utilised. Without any loss of generality, we can assume that a sufficiently high number of relays are roaming between the source and destination. We consider two approaches in order to choose the activated ones from the set of available relays ${ }^{1}$. The first approach is straightforward, while our novel proposition is based on ensuring that the appropriately shaped EXIT tunnel remains open:

${ }^{1}$ We can either choose two relays at an appropriate position or equivalently choose two relays at an arbitrary position, provided that appropriately controlled transmit powers are used at the source node and the two relay nodes. Two manners have the same influence on the system. For straightforwardness we follow the former.
1) We can simply pick two arbitrary relays that are about half-way between the $s$ node and the $d$ node.

2) The EXIT-chart based approach is that of choosing the optimal relays, which are those that facilitate the simulation creation of an open EXIT tunnel in Fig. 4 and Fig. 5, leading to the $(1,1)$ point at sufficiently high $S N R_{r}$ values at both the $r$ nodes and $d$ node, provided that the $S N R_{t}$ value is also sufficiently high. Denoting the difference between the two convergent points of two concatenated coding arrangements, namely those of the IrCC-URC and the IrCC-URC-STTC arrangements as $A(d B)$ or $a$ in terms of their ratio and using (4), we have :

$$
A=S N R_{r(\text { relay })}-S N R_{r(\text { dest. })}=G_{s r}-G_{r d} .
$$

Combining (10), (1), (2) and (3), we arrive at the optimal relayposition conditions as follows:

$$
\begin{gathered}
d_{s r}=\frac{d}{(1+\sqrt{a})}, d_{r d}=\frac{d \times \sqrt{a}}{(1+\sqrt{a})}, \\
g_{s r}=(1+\sqrt{a})^{2}, g_{r d}=\frac{(1+\sqrt{a})^{2}}{a} .
\end{gathered}
$$

The two EXIT charts of Fig. 4 and Fig. 5 provide $A=3.7 \mathrm{~dB}$. We then evaluate the path gains from (11), yielding $g_{s r}=6.41$ and $g_{r d}=2.73$ while the corresponding distances, $d_{s r}$ and $d_{r d}$, are evaluated from (1) and (2), which become $0.39 d$ and $0.61 d$, respectively.

\section{Simulation Results And Discussions}

In this section, we characterise the achievable BER versus $E_{b} / N_{0}$ performance corresponding to the two different relay-selection approaches in order to verify the results of our EXIT-chart aided design in Section IV. Although perfect relaying is not required for the success of our coding scheme, we produce benchmark results for the perfect-relaying scenario, where no errors are imposed by the source-relay link, in order to specify the achievable upper bound performance.

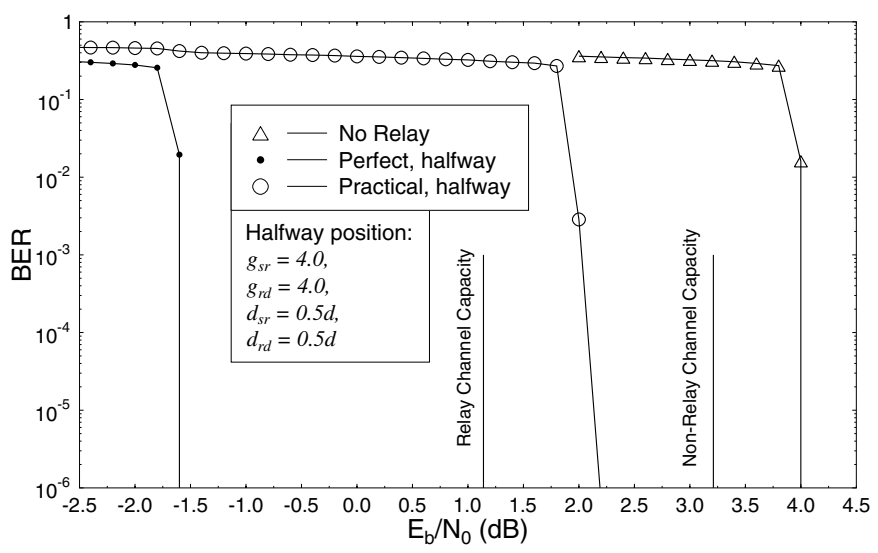

Fig. 6. BER performance, when the $r$ nodes are located half-way between the $s$ node and the $d$ node

As shown in Fig. 6, once utilising the relays at the half-way position, a $2.0 \mathrm{~dB} E_{b} / N_{0}$ improvement is achieved, when we compare the $E_{b} / N_{0}$ values at the BER of $10^{-5}$ between two scenarios, namely with and without the relays, which have $E_{b} / N_{0}$ values of $2.2 \mathrm{~dB}$ and $4.2 \mathrm{~dB}$, respectively. Note in Fig. 6 that the idealistic perfect relay scheme is operating beyond the actual relay channel capacity. As seen in Fig. 7, selecting the relays around the optimal position defined by (11) can provide a $4.0 \mathrm{~dB} E_{b} / N_{0}$ improvement at a $B E R$ of $10^{-5}$ in comparison to the scenario of no relaying, which is $2.0 \mathrm{~dB}$ better than that of relaying having the relays at the mid-way position. As 
seen in Fig. 6, the performance of the practical relay scheme is about $3.6 \mathrm{~dB}$ from that of the idealised perfect relay scheme, which is due to the effects of error propagation imposed by the relay nodes. More explicitly, the $S N R_{r \text { (relay) }}$ is too low to achieve a sufficiently low BER, although the $S N R_{r(\text { dest.) }}$ is sufficiently high to attain a low $\mathrm{BER}$, when the relay nodes are located half-way between the source and destination nodes. The optimal relay selection method aims to solve this problem by activating relays close to the optimal locations so that the system may reach the $S N R_{r \text { (relay) }}$ and $S N R_{r \text { (dest.) }}$ values required for simultaneously achieving a low BER at both the relay and destination nodes. The optimal relay nodes in the scheme considered are located at positions closer to the source node in order to achieve $g_{s r}=6.41$ and $g_{r d}=2.73$ according to (11). The optimal relay-aided performance seen in Fig.7 shows that the practical relaying scheme is now capable of operating a mere $0.2 \mathrm{~dB}$ away from the corresponding perfect relaying scheme.

Note that the performance gain of selecting relays at the optimum location, as opposed to using relays at the half-way location, will be either higher or lower when we have either a higher or a lower value for A, which quantifies the difference between the two convergence $S N R_{r}$ values given by (10). Fig. 6 and Fig. 7 also show that our coding scheme is capable of operating $1.1 \mathrm{~dB}$ and $1.1 \mathrm{~dB}$ away from the relay channel's capacity corresponding to the cases of mid-way relaying and optimal relaying, respectively. Note that relay channel's capacity, when employing the relays near the optimal position defined by (11), is always better than that of the relays near the midway position. (9) also suggests that there should be another optimal position for maximising the relay channel's capacity. However, our optimal condition defined in (11) always provide the best possible performance for our coding scheme regardless of the optimal position for maximising the relay channel's capacity.

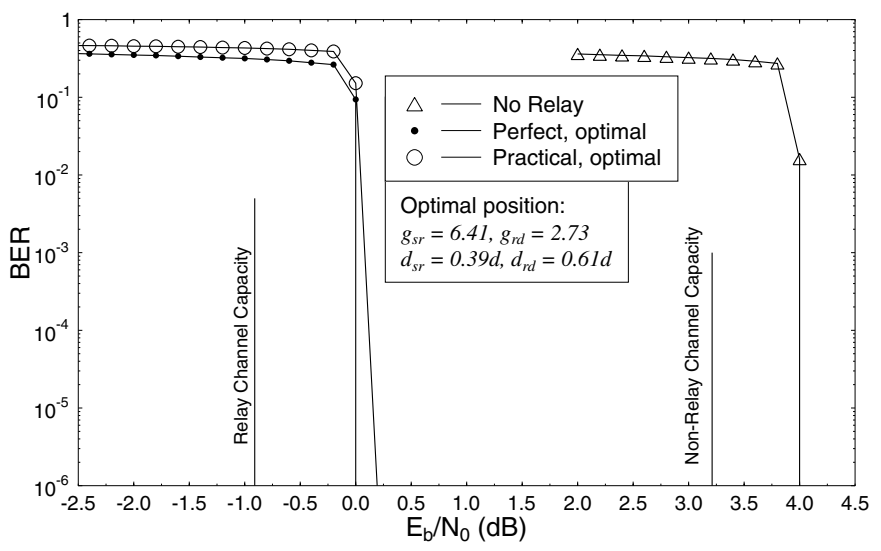

Fig. 7. BER performance, when selecting the $r$ nodes at the optimal position.

\section{CONCLUSIONS}

In this contribution, we have proposed a DC-IrCC-URC-STTC scheme for single-antenna based cooperative relaying. We formulated the associated near-capacity design principles relying on EXIT charts for arbitrary three-stage concatenated cooperative systems. In addition to that, in order to ensure our near-capacity coding scheme that it provides the best possible performance, we formulated and investigated an EXIT-chart based optimal condition for selecting the relays by ensuring that they are capable of maintaining an open EXIT chart tunnel. The simulation results verified the EXIT-chart aided code design and demonstrated that the DC-IrCC-URC-STTC scheme outperforms the non-cooperative IrCC-URC coding scheme by about $4.0 \mathrm{~dB}$ at a $B E R$ of $10^{-5}$. The optimal relay scheme also mitigated the problem of potential error propagation often imposed by the relays, which was achieved by selecting relay nodes near the optimal locations for ensuring that the received $S N R$ value required at the relay and destination nodes can be maintained simultaneously. The optimal relaying provided a $2.0 \mathrm{~dB}$ improvement in comparison to the case of mid-way relaying.

\section{REFERENCES}

[1] J. H. Winters, J. Salz, and R. D. Gitlin, "The impact of antenna diversity on the capacity of wireless communication systems," IEEE Transactions on Communications, vol. 42, pp. 1740-1751, Feb. 1994.

[2] V. Tarokh, N. Seshadri, and A. R. Calderbank, "Space-time codes for high data rate wireless communication: performance criterion and code construction," IEEE Transactions on Information Theory, vol. 44, pp. 744-765, Mar. 1998.

[3] S. M. Alamouti, "A simple transmit diversity technique for wireless communications," IEEE Journal on Selected Areas in Communications, vol. 16, pp. 1451-1458, Oct. 1998.

[4] V. Tarokh, A. Naguib, N. Seshadri, and A. R. Calderbank, "Space-time codes for high data rate wireless communication: performance criteria in the presence of channel estimation errors, mobility, and multiple paths," IEEE Transactions on Communications, vol. 47, pp. 199-207, Feb. 1999.

[5] M. C. Valenti and B. Zhao, "Distributed turbo codes: towards the capacity of the relay channel," in Proc. VTC 2003-Fall Vehicular Technology Conference 2003 IEEE 58th, vol. 1, pp. 322-326, Oct. 6-9, 2003.

[6] A. Sendonaris, E. Erkip, and B. Aazhang, "User cooperation diversity. Part I. System description," IEEE Transactions on Communications, vol. 51, pp. 1927-1938, Nov. 2003.

[7] J. N. Laneman, D. N. C. Tse, and G. W. Wornell, "Cooperative diversity in wireless networks: Efficient protocols and outage behavior," IEEE Transactions on Information Theory, vol. 50, pp. 3062-3080, Dec. 2004.

[8] A. Host-Madsen and J. Zhang, "Capacity bounds and power allocation for wireless relay channels," IEEE Transactions on Information Theory, vol. 51, pp. 2020-2040, June 2005.

[9] S. Yiu, R. Schober, and L. Lampe, "Distributed space-time block coding," IEEE Transactions on Communications, vol. 54, pp. 11951206, July 2006.

[10] S. ten Brink, "Convergence behavior of iteratively decoded parallel concatenated codes," IEEE Transactions on Communications, vol. 49, pp. 1727-1737, Oct. 2001.

[11] S. X. Ng, S. Das, J. Wang, and L. Hanzo, "Near-capacity iteratively decoded space-time block coding," in Proc. IEEE Vehicular Technology Conference VTC Spring 2008, pp. 590-594, May 11-14, 2008.

[12] M.Tücher and J. Hagenauer, "Exit charts of irregular codes," in Proceeding of the 36th Annual Conference on Information Sciences and Systems [CDROM], (Princeton, NJ, USA), Mar. 2002.

[13] S. ten Brink, "Rate one-half code for approaching the Shannon limit by 0.1 dB," Electronics Letters, vol. 36, pp. 1293-1294, July 20, 2000.

[14] R. Tee, R. G. Maunder, and L. Hanzo, "Exit-chart aided near-capacity irregular bit-interleaved coded modulation design," IEEE Transactions on Wireless Communications, vol. 8, pp. 32-37, Jan. 2009.

[15] M. Tuchler, "Design of serially concatenated systems depending on the block length," IEEE Transactions on Communications, vol. 52, pp. 209218, Feb. 2004.

[16] A. Ashikhmin, G. Kramer, and S. ten Brink, "Extrinsic information transfer functions: model and erasure channel properties," IEEE Transactions on Information Theory, vol. 50, pp. 2657-2673, Nov. 2004.

[17] M. Tuchler, "Convergence prediction for iterative decoding of threefold concatenated systems," in Proc. IEEE Global Telecommunications Conference GLOBECOM '02, vol. 2, pp. 1358-1362, Nov. 17-21, 2002.

[18] S. X. Ng, J. Wang, M. Tao, L.-L. Yang, and L. Hanzo, "Iteratively decoded variable length space-time coded modulation: Code construction and convergence analysis," IEEE Transactions on Wireless Communications, vol. 6, pp. 1953-1963, May 2007.

[19] L. Hanzo, O.Alamri, M. E.Hajjar and N.Wu, Near-Capacity MutiFunctional MIMO Systems. New York, USA : John Wiley and Sons, 2009.

[20] H. Ochiai, P. Mitran, and V. Tarokh, "Design and analysis of collaborative diversity protocols for wireless sensor networks," in Proc. VTC2004Fall Vehicular Technology Conference 2004 IEEE 60th, vol. 7, pp. 46454649, Sept. 26-29, 2004.

[21] S. X. Ng, J. Wang, and L. Hanzo, "Unveiling Near-Capacity Code Design: The Realization of Shannon's Communication Theory for MIMO Channels," in Proc. IEEE International Conference on Communications ICC '08, pp. 1415-1419, May 19-23, 2008. 